\title{
ANALYSIS OF BEAM-TO-UPRIGHT END CONNECTIONS STEEL STORAGE SYSTEMS
}

\author{
Lucas Alves Escanio, Guilherme Cássio Elias, Luiz Henrique de Almeida Neiva, \\ Vinícius Nicchio Alves and Arlene Maria Cunha Sarmanho*
}

\section{A B S T RA C T}

This study presents an experimental and numerical research into the behavior of beam-to-upright end connections, which are part of steel storage systems. A total of 21 cantilever tests comprising three upright and four beam cross sections, were conducted, and a finite element numerical model was developed. The model was developed to compare its results with experimental results and to extrapolate these results in a parametric analysis. The aim of the study is to evaluate the behavior, semi-rigidity, and influence of the geometric parameters in the stiffness of beam-upright lipped connections of pallet-type industrial storage systems. The results of the numerical model showed good correlation with the experimental results, in terms of moment-rotation behavior and the initial stiffness values, with a maximum difference of $10 \%$. In the parametric analysis, a pattern was observed in the variation of stiffness when the dimensions - mainly the height of beams-of the prototypes were increased.

\section{A R T I C LE H I S T O RY}

$\begin{array}{ll}\text { Received: } & 3 \text { October } 2019 \\ \text { Revised: } & \text { 22 June } 2020 \\ \text { Accepted: } & \text { 22 June } 2020\end{array}$

\section{K E Y W O R D S}

Cold-formed steel

Storage rack uprights;

Cantilever test;

Semi-rigid connection

Beam-to-upright lipped connection

\section{Introduction}

Industrial storage systems are traditionally used in various industries to store different types of manufactured products. These storage systems are produced in cold formed profiles. The uprights have sections specially developed to facilitate their assembly, containing perforations along their length to facilitate the connection with the beams. These systems provide great practicality, offering several configurations of storage arrangements considering the characteristics of each product. A rectangular hollow section is commonly used for the beams, and they are positioned in the structure perpendicular to the plane of the uprights with the purpose of receiving the load from the pallets and transmitting it to the uprights and bases by means of the connections

The connections between beams and uprights are semi-rigid. In the case studied in this work, the end connectors are formed by a plate containing lips, and they are placed at the beam's ends. The lips of the plate fit into the holes of the upright, which are evenly distributed along its length, to create unity between the upright and the beam [1]. The details of the beam and the lipped connector in this study are presented in Fig. 1 and Fig. 2.

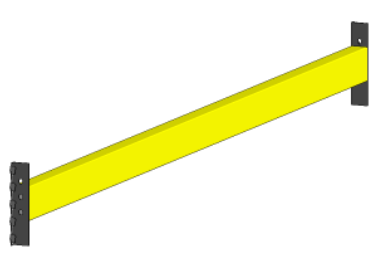

(a)

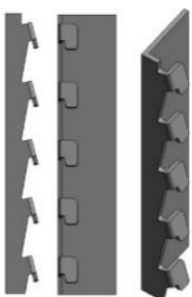

(b)
Fig. 1 (a) Beam with connectors; (b) The end connector studied

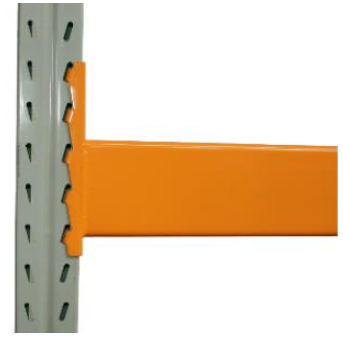

Fig. 2 Perforations and upright-beam connection

Shah et al. [2] performed an extensive study on state-of-the-art pallet rack connections of steel. The authors concluded that parameters such the height of the pallet beam, the column's thickness and the connector's geometry affects the strength and stiffness of the connector. Several numerical and experimental studies [3-5] have been carried out to investigate the influence of perforations on the upright's stability and load capacity.

Bajoria and Talikoti [6] determined the flexibility of beam-to-column connectors used in thin-walled cold-formed steel pallet racking systems by two different ways. The standard cantilever method and the proposed double cantilever method were used, and it was verified that to determine the flexibility of the connector, the double cantilever procedure can be used instead of the conventional cantilever test.

Based on experimental and numerical studies on the flexibility of the connection in cold formed steel racks, Prabha et al. [1] observed that the moment rotation characteristic of a connection is determined by the efficiency of the connection members (specially the upright) and by the design of the beam end connector. Furthermore, the increased height of the beam and thickness of the upright also increased the strength and stiffness of the joint.

Cardoso and Rasmussen [7] developed a finite element model to study storage rack frames, which used a bi or multi-linear moment rotation curve for the upright-pallet beam joints. They observed that the present component tests for rack frames connections overestimated the joint stiffness and were unable to emulate the behavior of the connection at the system level. The stiffness and semi-rigidity of the connector were also tested by Bajoria et al. [8], using the standard cantilever method and a double cantilever method. In addition, a finite element modal analysis of both tests was carried out. The proposed mathematical model was verified for single-, two-, and six-bay frames, with and without mass. All the frame results were very close to the free vibration modal analysis result. That means that this model could be used for any number of bays.

Several studies in the literature aim to study the cold-formed steel uprights and the connection between the upright and beams using experimental knowledge and numerical modeling [3,5,9-15].

In this study, the developed numerical model was calibrated with the experimental results to evaluate the behavior, semi-rigidity, and influence of the geometric parameters on the stiffness of beam-upright lipped connections of pallet-type industrial storage systems. Numerical and experimental studies were performed to determine the moment-rotation curve and connection stiffness. A total of 7 combinations of connection between beam and uprights with different cross-sections were tested in the experimental study. For each combination, 3 experimental tests were performed, resulting in 21 experimental tests.

A numerical finite element analysis of the 7 combinations was performed using the ANSYS software [16]. The numerical models were validated by comparing their behavior and stiffness with the experimental results, and the influence of each lip on the behavior of the connection was studied. A parametric study was carried out to evaluate the effects of the variation of the 
geometric parameters of the elements of the structural assembly on the connection's stiffness.

\section{Experimental program}

\subsection{Cross-section geometry}

A total of 21 cantilever tests [17] were conducted in this study to determine the stiffness and the bending strength of the lipped connection. Three upright and four beam cross sections were considered, totalizing seven combinations. The three different uprights considered for the cross section of the rack-type uprights had different web widths, $b_{w}=80,90$, and $100 \mathrm{~mm}$. The considered upright cross-sectional thickness was $t=2.00 \mathrm{~mm}$, and the cross-sections are shown in Fig. 3 with the dimensions in Table 1.

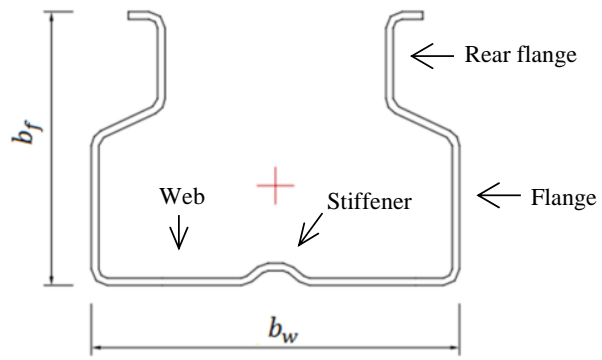

Fig. 3 Cross section considered for uprights with the elements identified

Table 1

Dimensions of upright cross section

\begin{tabular}{ccccc}
\hline Upright & Length $(\mathrm{mm})$ & $t(\mathrm{~mm})$ & $b_{w}(\mathrm{~mm})$ & $b_{f}(\mathrm{~mm})$ \\
\hline $\mathrm{U} 1$ & & 2.00 & 80 & 70 \\
$\mathrm{U} 2$ & 800 & 2.00 & 90 & 70 \\
$\mathrm{U} 3$ & & 2.00 & 100 & 74 \\
\hline
\end{tabular}

Based on the specifications of the European standard EN 15512:2009 [17], the length considered for the uprights was $L=800 \mathrm{~mm}$. The upright and its perforations are shown in Fig. 4.
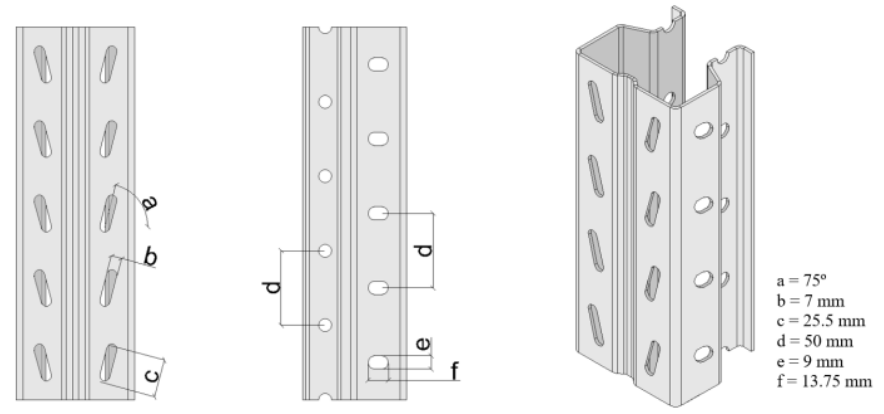

Fig. 4 Upright and perforations

Four rectangular hollow-section beams with different cross-sectional dimensions were considered, with different height $(h)$, width $(b)$, and thickness $(t)$, as presented in Fig. 5 and Table 2. Fig. 6 shows the cross-section properties of the studied uprights and beams.

The connection between the beams and the uprights is composed of five lips. The connection has the same dimension for all the prototypes tested. The lip dimensions are shown in Fig. 7. The prototypes tested were divided in three series, totalizing fifteen combinations of uprights and beams, which are listed in Table 3.

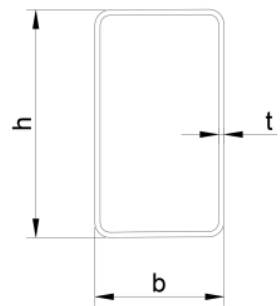

Fig. 5 Cross-section considered for beams

Table 2

Beam cross-section dimensions

\begin{tabular}{lcccc}
\hline Beam (type_t) & Length $(\mathrm{mm})$ & $b(\mathrm{~mm})$ & $h(\mathrm{~mm})$ & $t(\mathrm{~mm})$ \\
\hline B1_2.0 & 42 & 97 & 2.0 \\
B2_1.8 & 50 & 102 & 1.8 \\
\hline B2_2.0 & 50 & 102 & 2.0 \\
\hline B1 & 500 & 122 & \\
\hline
\end{tabular}

Fig. 6 Cross-section properties of the rack connection members (mm)

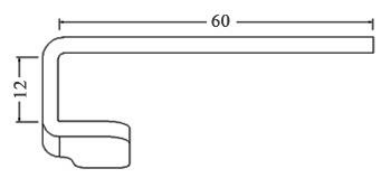

Fig. 7 Lip dimensions (mm)

Table 3

Series tested

\begin{tabular}{ccc}
\hline No. & Upright (type) & Beam (type_t $)$ \\
\hline \multirow{2}{*}{1} & $\mathrm{U} 1$ & B1_2.0 \\
& & B2_1.8 \\
& & B2_2.0 \\
2 & $\mathrm{U} 2$ & \\
& & B3_2.0 \\
\multirow{2}{*}{3} & $\mathrm{U} 3$ & B1_2.0 \\
& & B2_2.0 \\
& & B3_2.0 \\
\hline
\end{tabular}

\subsection{Material properties}

The upright and beam prototypes were fabricated from cold-formed steel. The values of yield stress $f_{y}$ and ultimate tensile strength $f_{u}$ were obtained from three tensile coupon tests, and the averaged results are presented in Table 4 . The tests reproduced the standard procedures for tensile testing presented in ABNT NBR 6892:2002 [18] 
Table 4

Tensile tests results.

\begin{tabular}{|c|c|c|c|}
\hline \multicolumn{2}{|c|}{ Prototype (type $\_t$ ) } & $f_{y}(\mathrm{MPa})$ & $\mathrm{f}_{\mathrm{u}}(\mathrm{MPa})$ \\
\hline \multirow{3}{*}{ Upright } & U1_2.0 & 391 & 524 \\
\hline & U2_2.0 & 380 & 517 \\
\hline & U3_2.0 & 371 & 523 \\
\hline \multirow{4}{*}{ Beam } & B1_2.0 & 399 & 516 \\
\hline & B2_1.8 & 329 & 460 \\
\hline & B2_2.0 & 397 & 518 \\
\hline & B3_2.0 & 397 & 518 \\
\hline
\end{tabular}

\subsection{Test assembly, instrumentation, and data acquisition}

The tests were performed based on the specification of the European standard EN 15512:2009 [17]. The schematic diagram of the test set-up is depicted in Fig. 8. The upright's ends simulated a fixed-end condition and were fixed to a reaction frame that was fixed in the reaction slab. One beam was connected to one column in each test. The displacements on the upper and lower connector's face were obtained by displacement transducers (linear variable differential transformers (LVDT1 and LVDT2 at Fig. 8)).

Another displacement transducer (LVDT3 at Fig. 8) was positioned to obtain the vertical displacement under the loading applied by a jack. The measurements got by the displacement transducers (LVDTs) and the data were registered on an HBM Spider8 automatic data acquisition system. The ultimate moment capacity on the connections was calculated with the load applied by the jack and measured by the load cell. The connection stiffness was calculated based on the RMI [19] specifications. The cantilever type test set-up arrangement is shown in Fig. 9.

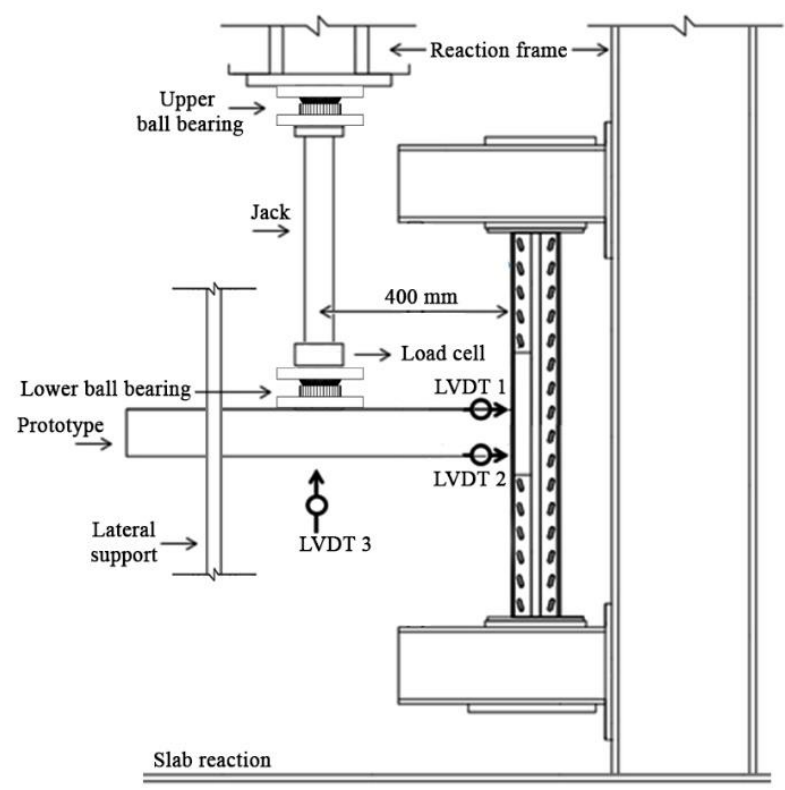

Fig. 8 Schematic diagram of the test set-up

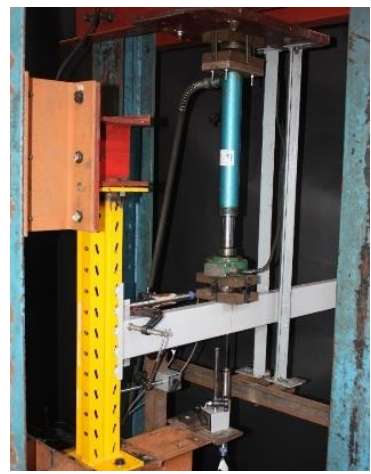

(a)

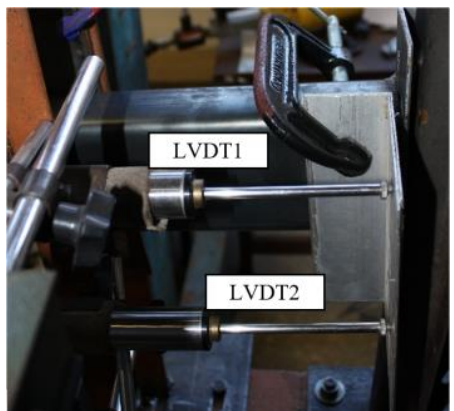

(b)

Fig. 9 Cantilever test set-up: (a) scheme and (b) position of LVDT1 and LVDT2

\subsection{Discussion on the test results}

The ultimate moment capacity on the connections was calculated by multiplying the load value applied by the jack and measured by the load cell by the distance of $400 \mathrm{~mm}$. The rotation $\theta$ at the ultimate moment was calculated using Eq. 1.

$\theta=\frac{\delta_{2}-\delta_{1}}{d}$

where:

$\delta_{1}, \delta_{2}$ are the deflections measured by LVDT1 and LVDT2;

$d$ is the distance between LVDT1 and LVDT2, and it equals the beam's height.

The averaged results of the failure load, ultimate moment capacity, and rotation at the ultimate moment in radians are shown in Table 5.

Table 5

Strength of the connector.

\begin{tabular}{cccc}
\hline Prototype & Failure load $(\mathrm{kN})$ & Ultimate moment & Rotation at the ultimate \\
\hline U1_2.0 $\times$ B1_2.0 & 4.28 & 1.71 & 0.064 \\
U1_2.0 $\times$ B2_1.8 & 4.24 & 1.71 & 0.057 \\
U1_2.0 $\times$ B2_2.0 & 4.34 & 1.74 & 0.059 \\
U2_2.0 $\times$ B3_2.0 & 5.43 & 2.17 & 0.051 \\
U3_2.0 $\times$ B1_2.0 & 4.64 & 1.86 & 0.056 \\
U3_2.0 $\times$ B2_2.0 & 4.47 & 1.79 & 0.061 \\
U3_2.0 $\times$ B3_2.0 & 5.41 & 2.06 & 0.049 \\
\hline
\end{tabular}

The initial stiffness of each experimental test and the respective average are shown in Table 7.

\section{Numerical Analysis}

A numerical study of 7 models was conducted to obtain reliable results of strength and stiffness for other arrangements of upright and beam cross sections. The ANSYS finite element software [16] was used to model the upright, beam, and connector with the five lipped connectors. The geometry, boundary, and loading conditions of the finite element model were developed to simulate the cantilever test, as shown in Fig. 10, with the same dimensions and details of the test prototypes and the material properties from the tensile tests results. The Young's modulus and Poisson's ratio considered for all members were $200 \mathrm{GPa}$ and 0.3 , respectively. The beam was connected to the upright using a connection with five lips (Fig. 11). 


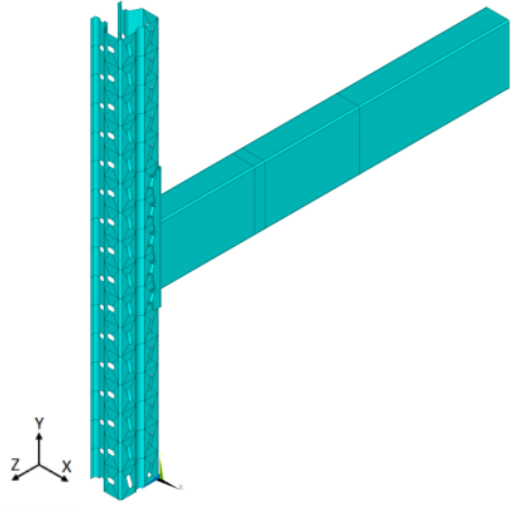

Fig. 10 Finite element model of the beam-upright connection

Geometric nonlinearity was considered adopting the full Newton Raphson solution method, and to simulate the physical nonlinearity, a multilinear model (multilinear isotropic hardening) with three points representing the stress-strain curve was adopted [20]. To solve the systems of linear equations, the sparse solver method was applied, which optimizes the process by reducing the number of operations required to solve the systems.

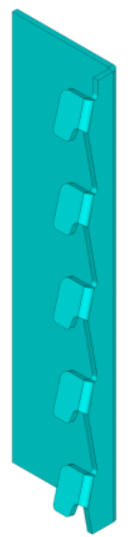

Fig. 11 Five lipped connectors

\subsection{Elements and mesh refinement}

The finite element used in the connector was SOLID45, which provided satisfactory results. It is an eight-node element with three degrees of freedom at each node, with plastic capacity. For the uprights and beams, the element used was SHELL181 [21], a four-node element with six degrees of freedom by node. To consider the contact between element, surface-to-surface contact elements [6], CONTA174 and TARGE170 were considered. Table 6 shows all the pairs used on the model.

\section{Table 6}

Tensile tests results.

\begin{tabular}{ccc}
\hline Contact & CONTA174 & TARGE170 \\
\hline Connector and upright & Upright's flange and web & Connector's plates \\
Lip and upright & Web perforation node lines & Surface of each lip \\
Beam and connector & Lateral beam nodes & Connector surface plate \\
\hline
\end{tabular}

Mesh sizes of 4, 5, and $6 \mathrm{~mm}$ were tested in the upright columns (Fig. 12a). The numerical results were compared to the experimental results and a better correlation was observed for the size of $5 \mathrm{~mm}$. In a similar way (Fig. 12b), the same study was performed on the beams for mesh sizes of 6,8 , and $10 \mathrm{~mm}$. In the beams, the mesh size was of $8 \mathrm{~mm}$. The mesh used in the analysis is presented in Fig. 13. On the interface of the solid-shell elements, a large stress concentration was observed, and $4 \mathrm{~mm}$ sides were used on the beam connector region to reduce this effect. Another study was conducted on the interface of the solid-shell elements regarding the number of layers in the solid element thickness, with 3, 4, and 5 layers. The results were very similar (Fig. 14). Therefore, the lowest value (3 layers) was used, because it presented a shorter processing time.

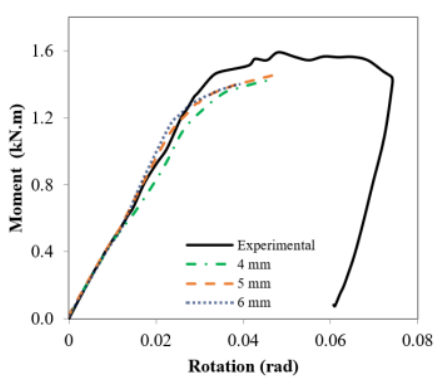

(a)

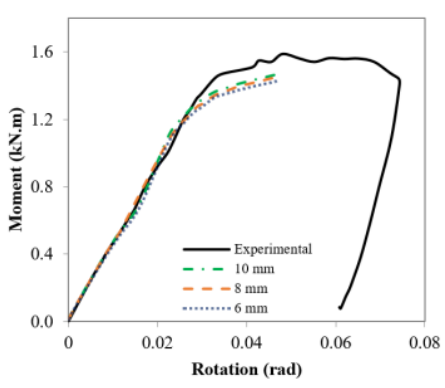

(b)
Fig. 12 Mesh study on prototype U1_2.0 × B1_2.0: (a) upright; (b) beam

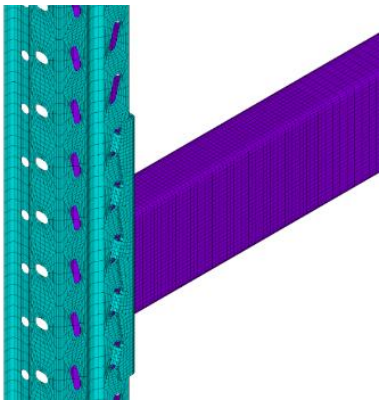

Fig. 13 Mesh used in ANSYS

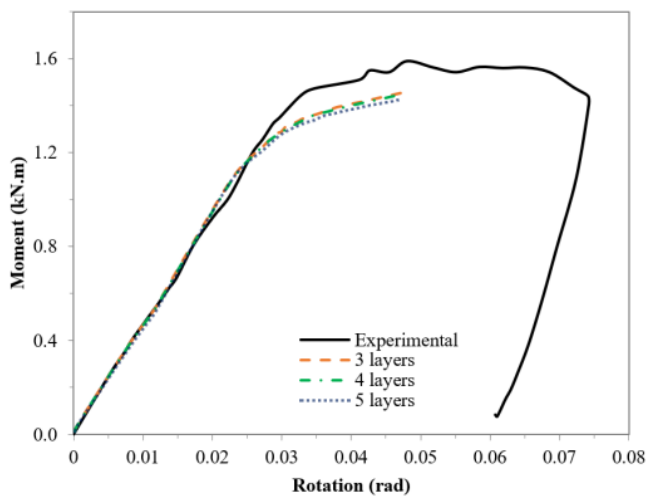

Fig. 14 Influence of number of layers on solid elements

\subsection{Boundary conditions}

The boundary conditions replicate the test assembly. The upper and lower upright end nodes had their rotations and translations restrained in all directions, simulating a fixed upright (see Fig. 15). The beams' lateral displacement was prevented by lateral displacement nodes stopped in the $\mathrm{x}$ direction (Fig. 16), which simulated the lateral support of the tests (Fig. 8). Their objective was to prevent lateral displacement of the beam.

The simulation of load application on the beam was performed incrementally, applying a displacement at the top of the beam (Fig. 16), in the position performed in the tests. The nodes of these lines were coupled, and a 20 mm-displacement was applied. An initial increment of $0.5 \mathrm{~mm}$ was defined, ranging from $0.1 \mathrm{~mm}$ to $1.0 \mathrm{~mm}$, according to the need evaluated automatically by Ansys.

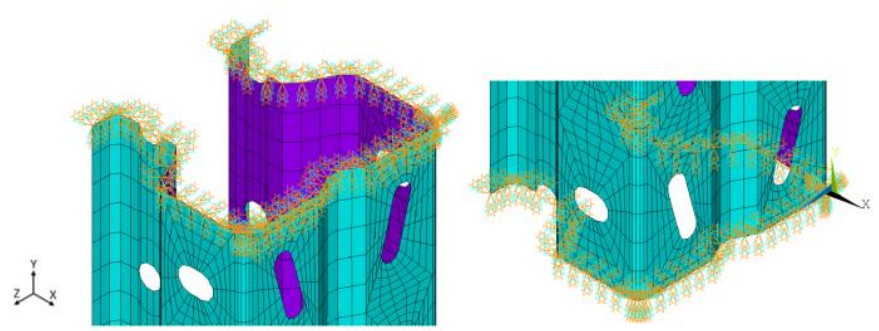

Fig. 15 Upright boundary conditions 


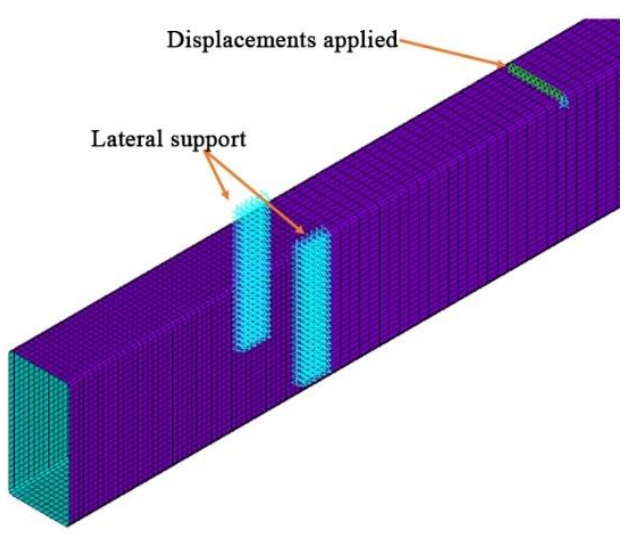

Fig. 16 Beam boundary conditions and load application

\subsection{Numerical results}

\subsubsection{Moment versus rotation curves}

The moment and rotation curves $(\mathrm{M}-\theta)$ of the numerical and experimental models were compared. In the numerical model, the displacement was gradually applied at the top of the beam, and the corresponding rotation, $\theta$, was calculated with Eq. 1 using the displacements of the $\mathrm{Z}$-axis of the nodes N1 and N2, as shown in Fig. 17.

The comparisons of the moment and rotation curves of the numerical and experimental models are presented in Fig. 18. The curves had a non-linear behavior. A satisfactory correlation was observed between the numerical and experimental curves. The important factors that influence this non-linearity are the slippage between the lipped connectors and the upright perforations, yielding of some regions of the lips due to a localized stress concentration effect, and geometrical imperfections in the tested prototypes. The observed differences in the experimental results of the same prototype were due to existent imperfections in the specimens.
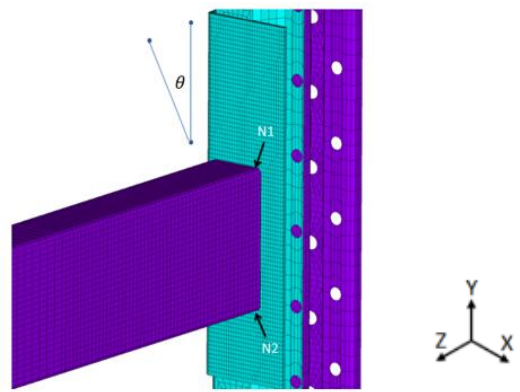

Fig. 17 Monitored nodes to measure rotation

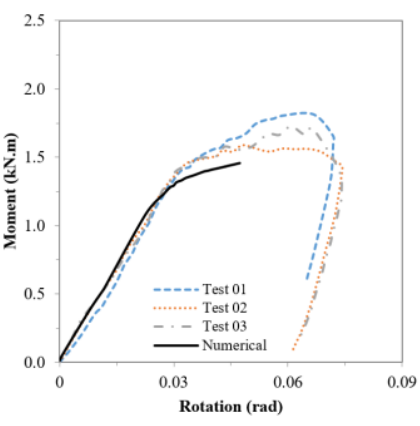

(a) U1_2.0 $\times$ B1_2.0

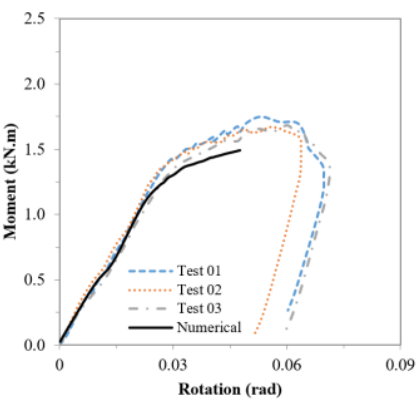

(b) $\mathrm{U} 1 \_2.0 \times$ B2__1.8

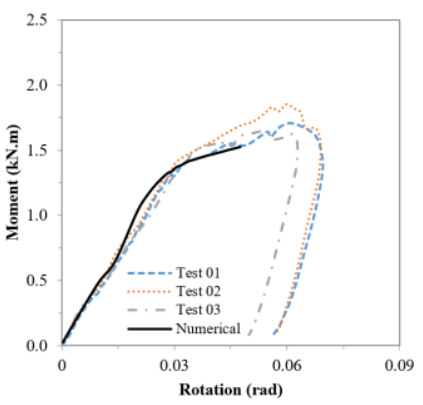

(c) U1_2.0 $\times$ B2_2.0

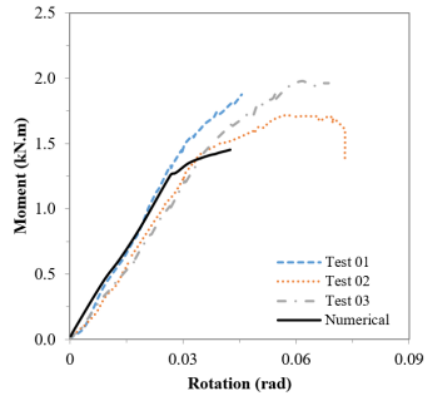

(e) $\mathrm{U}_{-} 2.0 \times$ B1_ 2.0

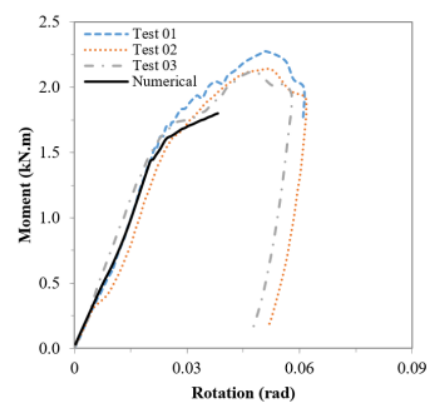

(d) $\mathrm{U} 2.2 .0 \times \mathrm{B} 32.0$

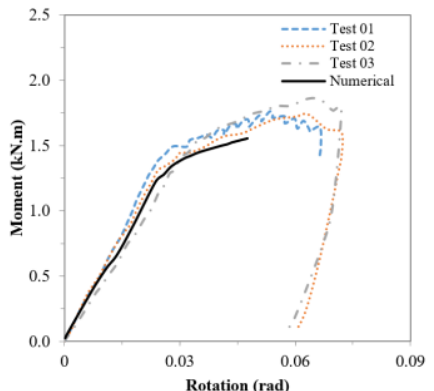

(f) $\mathrm{U} 3 \_2.0 \times$ B2_2.0

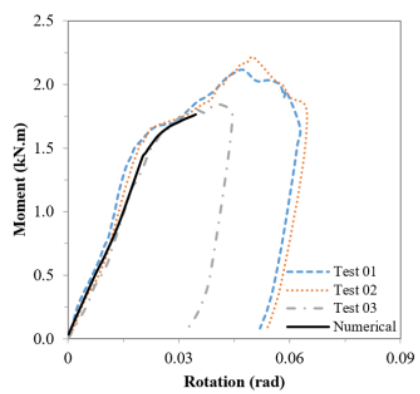

(g) $\mathrm{U}_{-} 2.0 \times \mathrm{B} 3{ }_{-} 2.0$

Fig. 18 Comparison of the numerical and experimental moment $\times$ rotation curves

\subsubsection{Comparisons of initial stiffnes}

The experimental and numerical initial stiffness of the models were compared. These values are presented in Table 7. The numerical ultimate moment capacity was always less than the experimental ultimate moment capacity. Therefore, the initial stiffness method was used to compute the connection stiffness. From the analysis of the results of stiffness of the experimental tests, it was concluded that some prototypes with the same dimensions presented significant differences in stiffness values. Again, there are significant influences of the imperfections in the results. The average stiffness of the numerical models had a good correlation with the average stiffness of the experimental analysis, presenting a maximum difference of $10 \%$.

Table 7

Comparison of experimental and numerical initial stiffness.

\begin{tabular}{ccccccc}
\hline & \multicolumn{3}{c}{ EXP } & \multicolumn{2}{c}{ NUM } & \\
\cline { 2 - 5 } Prototype & Test 1 & Test 2 & Test 3 & Average & Init. Stiff. & Dif (\%) \\
\hline U1_2.0 $\times$ B1_2.0 & 43.50 & 45.46 & 45.82 & 44.93 & 47.81 & 6.0 \\
U1_2.0 $\times$ B2_1.8 & 49.86 & 51.14 & 45.95 & 48.98 & 48.33 & 1.3 \\
U1_2.0 $\times$ B2_2.0 & 45.04 & 47.65 & 43.75 & 45.48 & 50.45 & 9.9 \\
U2_2.0 $\times$ B3_2.0 & 70.96 & 61.38 & 73.13 & 68.49 & 70.89 & 3.4 \\
U3_2.0 $\times$ B1_2.0 & 48.83 & 41.04 & 39.31 & 43.06 & 47.24 & 8.8 \\
U3_2.0 $\times$ B2_2.0 & 58.24 & 53.89 & 46.60 & 52.91 & 51.51 & 2.6 \\
U3_2.0 $\times$ B3_2.0 & 84.10 & 78.37 & 66.82 & 76.43 & 70.87 & 7.3 \\
\hline
\end{tabular}

After an analysis of the results, it was concluded that the moment versus rotation curves of the numerical model and experiments had similar behaviors, 
with the same formats in the linear section, and the stiffness of the numerical model was compatible with the stiffness of the experiments. Fig. 19 presents a schematic representation of the stiffness values of the numerical model and the experimental average stiffness. Considering the calculation of the initial stiffness, the results obtained with the proposed numerical model had values compatible with the experimental data. This indicates that the model can predict with good reliability the initial stiffness of the connection.

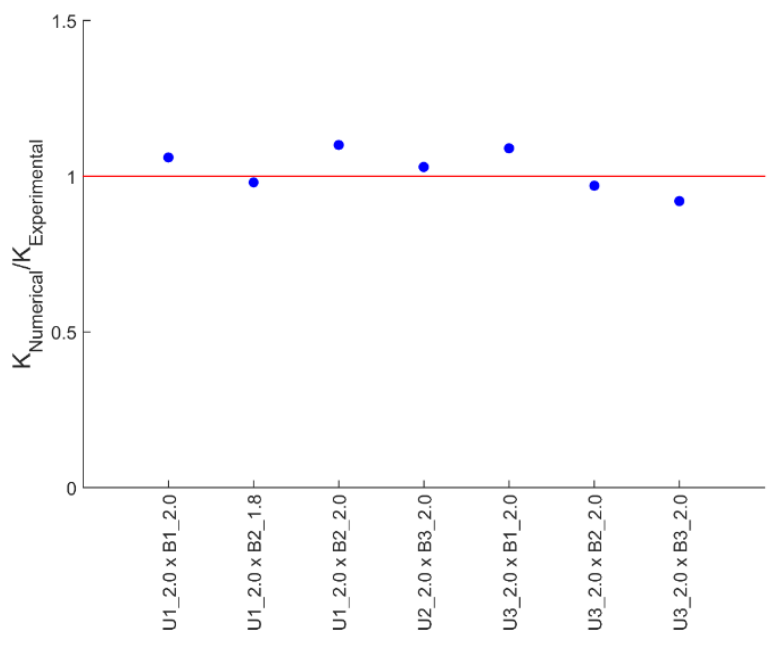

Fig. 19 Comparison of numerical and experimental stiffness

\section{Failure modes}

As observed in the analysis of the curves $(\mathrm{M}-\theta)$ of the experimental tests, there was a linear behavior from the beginning of the experiment. Imperfections and slack in the prototypes accentuated this behavior. In the numerical model, it was also possible to notice this behavior in the linear part of the curve. A study was carried out to identify the causes of this phenomenon in the substep of the numerical model where this behavior occurred.

The differences between the three types of beam (B1, B2, and B3) refer to the height and width of the cross section. Thus, the lips' solicitation will be different on each type. One model corresponding to each beam type was analyzed, as follows: (U3_2.0 $\times$ B1_2.0), (U3_2.0 × B2_2.0), and (U3_2.0 $\times$ B3_2.0).

It was identified that the displacements in the lipped connector had great influence on the changes in inclination in the initial part of the curve $(\mathrm{M}-\theta)$. Thus, these displacements were correlated with the points of the curve (M- $\theta)$ in which the model lost linearity. The displacement on the z-axis of the lipped connector was monitored, and it was identified as illustrated in Fig. 20.

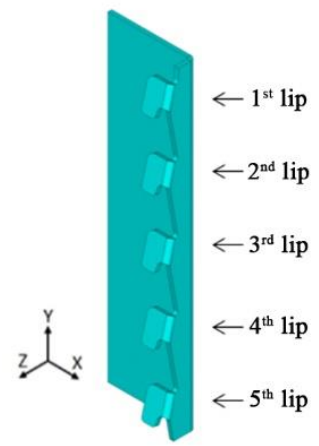

Fig. 20 Identification of lips and coordinate axes

The first analyzed model was U3_2.0 × B1_2.0. The M- $\theta$ curve of the experimental and numerical tests is shown in Fig. 21 . The vertical lines indicate points where the numerical model changes the slope of the curve. The first vertical line indicates a change in slope and a decrease in the rate of change in the $\mathrm{M}-\theta$ curve; the second one indicates another change in slope and an increase in the rate of change, and the third vertical line shows a decrease in the rate of change in $\mathrm{M}-\theta$ curve. The fourth vertical line indicates a sharp change in slope and loss of linearity of the numerical model. The displacement in each lip of the connector versus rotation is shown in Fig. 22. The vertical lines indicate points where the lips can change their behavior during the analysis.

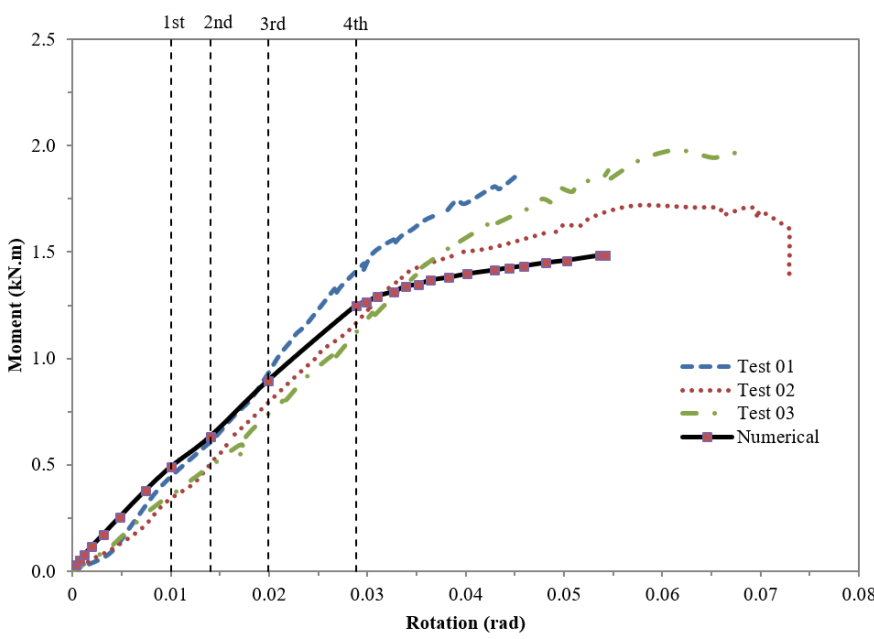

Fig. 21 Moment versus rotation of U3_2.0 $\times$ B1_2.0 model

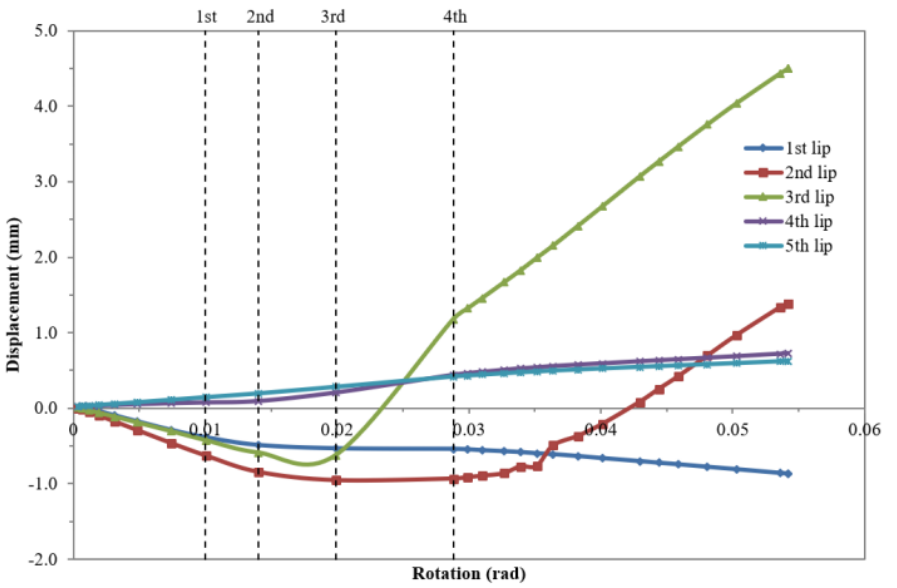

Fig. 22 Displacement versus rotation of each lip of U3_2.0 $\times$ B1_2.0 model

Note that the fourth and fifth lips do not contribute significantly in the resistance to rotation of the connection because their displacements are always positive in the z-axis, which is due to the compression force of the binary of the moment that appears in the base of the beam. Analyzing the von Mises stress in the numerical model in the substep of the second vertical line, the beginning of the yield at the base of the second and third lip is observed, and an increase in the slope of the curve $(\mathrm{M}-\theta)$ occurs owing to the absorption of the energy of the loading by these lips. It was observed a decrease in slope of the curve (M- $-\theta)$ from the third vertical line. This is because the third lip presents the entire section of its fold in a plastic state, no longer absorbing the energy of the load and only moving in the positive direction of the z-axis. The fourth vertical line marks the beginning of the loss of nonlinearity of the numerical model, coinciding with the beginning of the displacement in the positive direction of the z-axis of the second lip, because the section of its fold is already in a fully plastic state.

The collapse of the lipped connection occurred when there was an excessive deformation in the second lip, as can be seen in Fig. 23a. It is noted that the excessive displacement in the second lip did not occur in the numerical model (Fig. 23b), because it did not reach the rupture point.

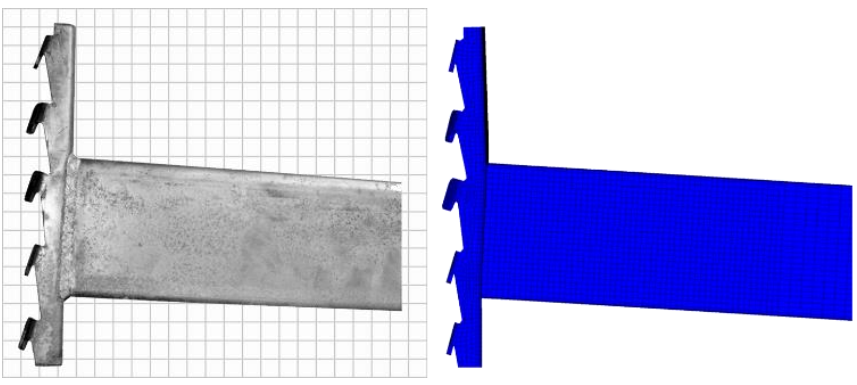

Fig. 23 Displacements on the lipped connection U3_2.0 × B1_2.0 model: (a) experimental; (b) numerical 
For the model U3_2.0 $\times$ B2_2.0, the moment versus rotation curve is shown in Fig. 24 and the displacement on each lip of the connector versus rotation is shown in Fig. 25. As in the previous analysis, the vertical lines helped to identify the points where the numerical model changed the slope of the curve and showed points where the lips changed their behavior during the analysis. As in the other model, the fourth and fifth lips did not contribute significantly to the resistance to rotation of the connection. In the substep corresponding to the third vertical line, the plastic deformation of the second and third lip begins. In the substep of the fourth vertical line, the section of the fold of the third lip is in a fully plastic state. Note that from the fifth vertical straight line, the model begins to lose linearity, coinciding with a change in the slope of the first lip, which can be characterized by the return of the slip. Small variations occur in the slope of the curve (M- $\theta$ ), until finally the section of the second lip fold is in a fully plastic state and starts to move in the positive $\mathrm{z}$-axis direction, causing the model to lose the non-linearity completely.

In none of the analyzed numerical prototypes, the model lost its linearity completely, and there was yield of the other elements that make up the connection, upright and beam, as can be seen in Fig. 26. Yield of the uprights did not occur during the entire analysis, and the beam had only a point of yield in the last loading step of the analysis.

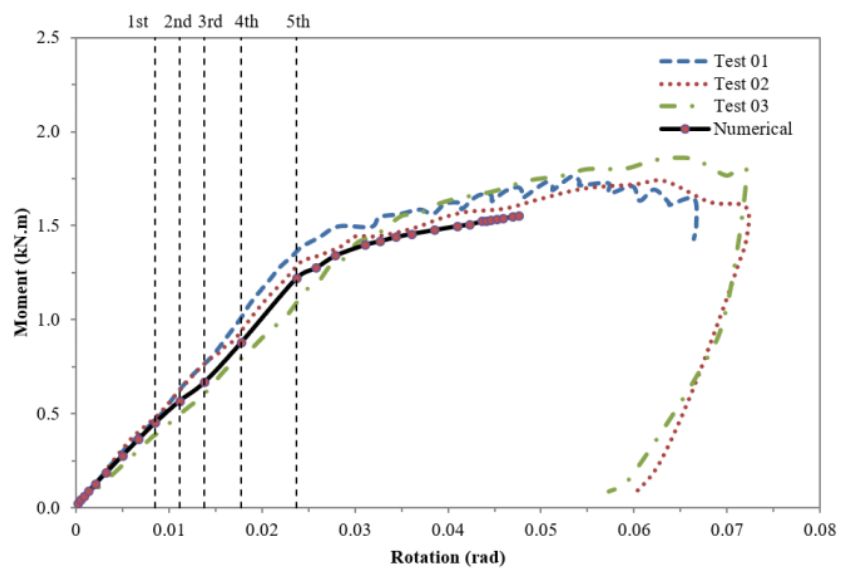

Fig. 24 Moment versus rotation of U3_2.0 $\times$ B2 _2.0 model

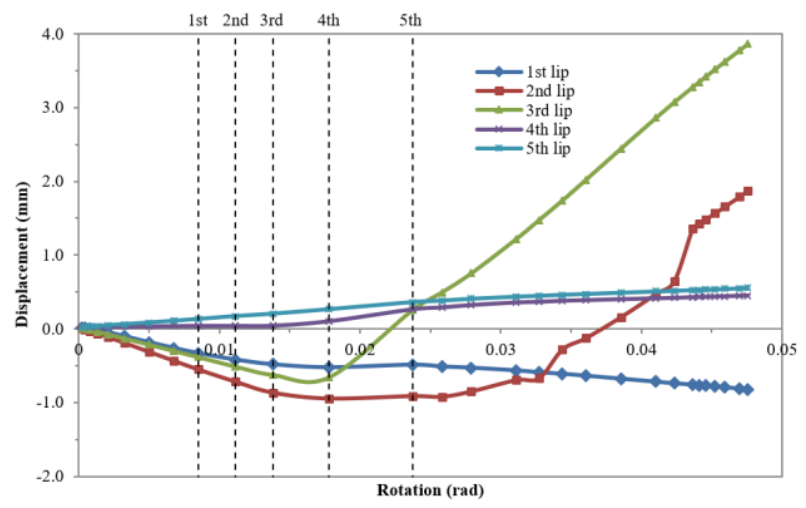

Fig. 25 Displacement versus rotation of each lip of U3_2.0 $\times$ B2_2.0 model

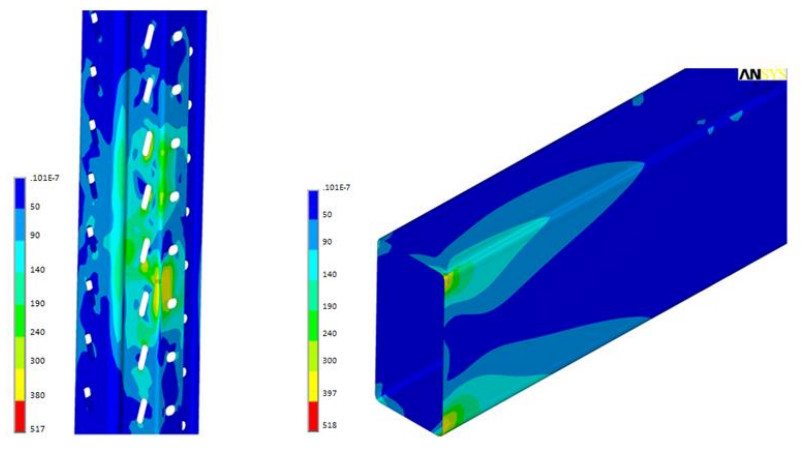

Fig. 26 von Mises stress of U3_2.0 × B2_2.0 model: (a) upright; (b) beam in Fig. 27, and the displacement on each lip versus rotation is shown in Fig. 28 A similar behavior is observed on the curves.

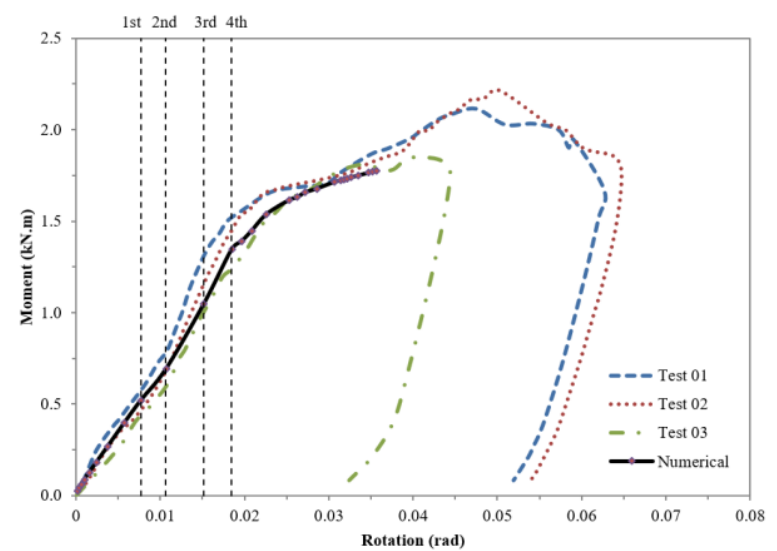

Fig. 27 Moment versus rotation of U3_2.0 × B3_2.0 model

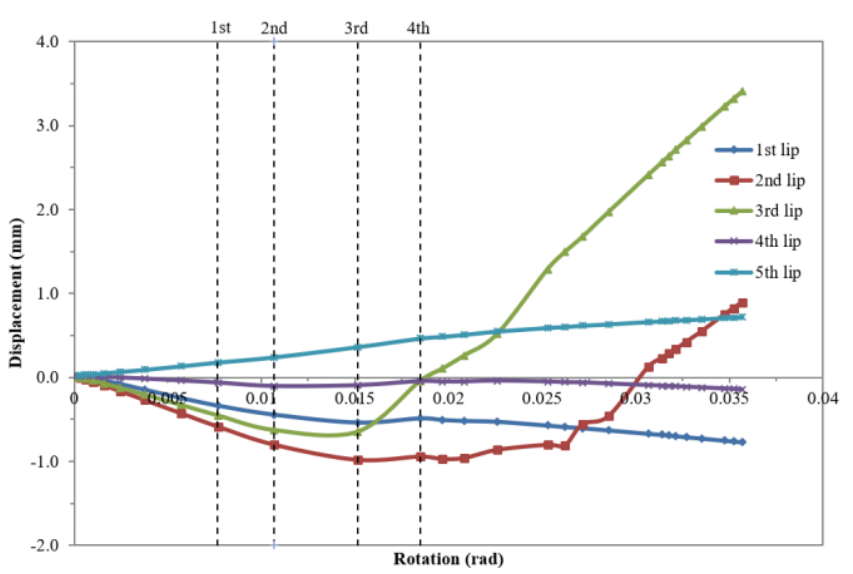

Fig. 28 Displacement versus rotation of each lip of U3_2.0 $\times$ B3_2.0 model

It was observed that, for this model, there was a greater contribution of the fourth lip in the resistance to rotation of the connection, whereas the fifth lip continued without a significant contribution. It can be noticed that the changes in the inclination of the curve M- $\theta$ are smoother. This is because the lips work together, without overloading any of them individually. At the starting point of the third vertical line (Fig. 28) the section of the third lip was already in a fully plastic state. As the first, second, and fourth lips are absorbing the energy of the loading, even with the beginning of the displacement of the third lip in the positive direction of the $\mathrm{z}$-axis, there is no change in slope of the curve M- $\theta$. From the fourth vertical line (Fig. 28) the second lip section is in an almost fully plastic state, but different from the other analyses, a contribution of the fifth lip in the resistance to rotation does not allow the second lip to be overloaded. The model continues with smooth changes in the slope of the curve M- $\theta$ until the entire section of the second lip is in a plastic state and begins its displacement in the positive direction of the $\mathrm{z}$-axis, thus resulting in a complete loss of linearity of the model.

The first and second models analyzed show similar lip behavior. The third model was different, with a greater contribution of the fourth lip in the resistance. This can be explained by the beam cross section. The first two have similar heights (97 and $102 \mathrm{~mm}$, respectively), and the last one is the highest (122 mm).

\section{Parametric analysis}

To better understand the influence of the geometric parameters on the connection stiffness in pallet rack structures, a parametric study was conducted with the models developed in the numerical analysis. The analysis was performed by changing the dimensions of the prototypes with commercial configurations. In total, 99 models were made with different combinations of beam and upright dimensions with the same connector's dimensions, and the stiffness values are depicted in Fig. 29. 


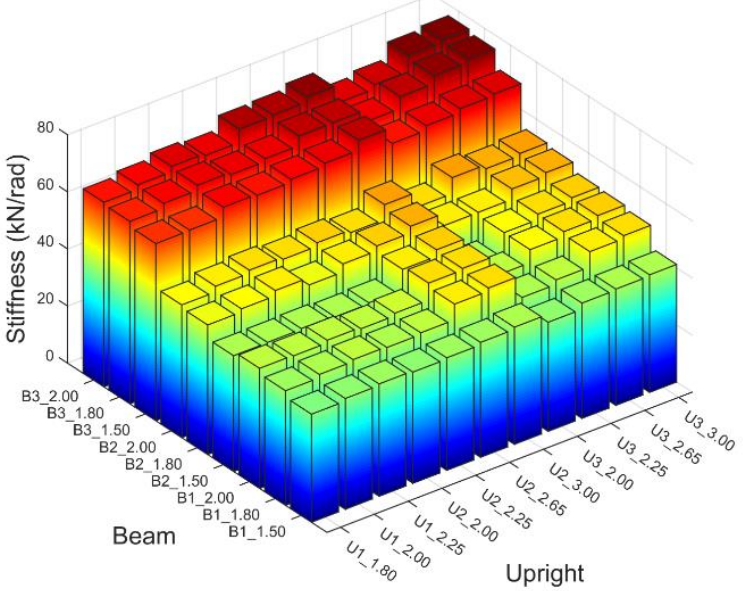

Fig. 29 Stiffness of parametric analysis

It was noted that by varying the dimensions of the beams while keeping the upright dimensions fixed, and then varying the beam dimensions and maintaining the upright dimensions fixed, a pattern of stiffness variation is observed. By varying the upright's web weight (grouped by types U1, U2, and U3) and maintaining its thickness fixed, the stiffness values of the models were compared. The largest variation observed was $4.24 \%$, when changing the size from U2 2.25 to U3 2.25. For this configuration, there is a $26 \%$ increase in moment of inertia in the axis of greater inertia.

By varying the thickness for the same upright type, it could be noticed that for type U1 and type U3, the greatest variation in stiffness occurred when there was a change in thickness from 2.0 to $2.25 \mathrm{~mm}$, presenting an increase of $2.68 \%$ and $6.34 \%$, respectively. For type U2, the greatest variation in stiffness occurred when the thickness changed from 2.25 to $2.65 \mathrm{~mm}$. In addition to the increase in moment of inertia, the increase in thickness of the upright generated a greater area of contact between the connector's lips and the holes of the upright.

By maintaining fixed the dimensions of the beam and changing its thickness, it was noticed that the greatest variations in stiffness occurred when the thickness changed from 1.5 to $1.8 \mathrm{~mm}$ for all types of beams, B1, B2, and B3, which presented an increase in stiffness of $5.78 \%, 9.32 \%$, and $1.70 \%$, respectively. With a variation in the cross-sectional dimensions of the beam (base and height), the greatest variation in stiffness occurred with the change from B2_1.5 to B3_1.5, generating an increase in stiffness of $32.91 \%$.

\section{Conclusions}

The objective of this work was the study of the connections in pallet storage systems fabricated in cold formed profiles, through experimental, numerical, and parametric analyses. The numerical model developed with the finite element method presented results with good correlation with those obtained by the experimental tests. For instance, the maximum difference between the numerical and experimental values of initial stiffness was $10 \%$. It was noticed that the behavior of the moment versus rotation curve of the experimental prototypes showed good correlation with the numerical values for the initial loading levels, with higher dispersion occurring from the beginning of the yield. Hence, the initial stiffness values were considered.

From the parametric analysis, a pattern was observed in the variation of stiffness when the dimensions of the prototypes were increased. The greatest variation was of $32.91 \%$, and resulted from increasing the height of the beam in $19.6 \%$.

The loss of linearity in the initial sections of the moment versus rotation curves, which occurred in the experimental tests, was caused by the variation in the displacements of the connector lips. It was noted that the third lip was the first to reach the yield stress and the first to generate displacement in the positive direction of the $\mathrm{z}$-axis. This displacement was observed in both the numerical and experimental analyses. The complete loss of non-linearity of the numerical model occurred when the section of the second lip fold was in a plastic state and began to move until the connection collapsed.

Finally, it was noticed that the upright and beam did not reach the yield stress at any point in the linear stretch of the moment versus rotation curve. To the point where the numerical model was able to reproduce the behavior of the connection, corresponding to a load that generated approximately $20 \mathrm{~mm}$ of displacement at the upper end of the beam, the upright did not reach the yield stress at any point. In other words, all the failure and collapse mechanisms occurred exclusively in the end connector.

\section{Acknowledgment}

The authors acknowledge the support provided by the National Council for Scientific and Technological Development (CNPq), Coordination of Improvement of Higher Level Personnel (CAPES), Minas Gerais State Research Support Foundation (FAPEMIG), Federal University of Ouro Preto (UFOP) and Águia Systems.

\section{References}

[1] Prabha P, Marimuthu V, Saravanan M, Arul Jayachandran S. Evaluation of connection flexibility in cold formed steel racks. J Constr Steel Res 2010;66:863-72. doi:10.1016/j.jcsr.2010.01.019.

[2] Shah SNR, Sulong NHR, Jumaat MZ, Shariati M. State-of-the-art review on the design and performance of steel pallet rack connections. Eng Fail Anal 2016;66:240-58 doi:10.1016/j.engfailanal.2016.04.017.

[3] Neiva LH de A, Sarmanho AMC, Faria VO, Souza FT de, Starlino JAB. Numerical and experimental analysis of perforated rack members under compression. Thin-Walled Struct 2018;130:176-93. doi:10.1016/j.tws.2018.05.024.

[4] Casafont M, Magdalena Pastor M, Roure F, Peköz T. An experimental investigation of distortional buckling of steel storage rack columns. Thin-Walled Struct 2011;49:933-46. doi:10.1016/j.tws.2011.03.016.

[5] Elias GC, Neiva LH de A, Sarmanho AMC, Alves VN, Castro AFB. Ultimate load of steel storage systems uprights. Eng Struct 2018;170:53-62. doi:10.1016/j.engstruct.2018.05.078.

[6] Bajoria KM, Talikoti RS. Determination of flexibility of beam-to-column connectors used in thin walled cold-formed steel pallet racking systems. Thin-Walled Struct 2006;44:372-80. doi:10.1016/j.tws.2006.01.007.

[7] Sena Cardoso F, Rasmussen KJR. Finite element (FE) modelling of storage rack frames. J Constr Steel Res 2016;126:1-14. doi:10.1016/j.jcsr.2016.06.015.

[8] Bajoria KM, Sangle KK, Talicotti RS. Modal analysis of cold-formed pallet rack structures with semi-rigid connections. J Constr Steel Res 2010;66:428-41. doi:10.1016/j.jcsr.2009.10.005.

[9] Markazi FD, Beale RG, Godley MHR. Experimental analysis of semi-rigid boltles connectors. Thin-Walled Struct 1997;28:57-87. doi:10.1016/s0263-8231(97)00003-7.

[10] Zhao X, Wang T, Chen Y, Sivakumaran KS. Flexural behavior of steel storage rack beamto-upright connections. J Constr Steel Res 2014;99:161-75. doi:10.1016/j.jcsr.2014.04.007.

[11] Aguirre C. Seismic behavior of rack structures. J Constr Steel Res 2005;61:607-24. doi:10.1016/j.jcsr.2004.10.001

[12] Escanio LA. Análise numérica de ligações em sistemas de armazenagem industrial. Master Dissertation, Ouro Preto: 2019

[13] Freitas AMS, Freitas MSR, Souza FT. Analysis of steel storage rack columns. J Constr Steel Res 2005;61:1135-46. doi:10.1016/j.jcsr.2005.02.004

[14] Freitas AMS, Freitas MSR, Souza FT, Silva GG, Faria VO. Theoretical and experimenta analysis of perforated rack columns. Rev Esc Minas 2013;66:289-94. doi:10.1590/S037044672013000300004

[15] Gusella F, Arwade SR, Orlando M, Peterman KD. Influence of mechanical and geometric uncertainty on rack connection structural response. J Constr Steel Res 2019;153:343-55. doi:10.1016/j.jcsr.2018.10.021

[16] ANSYS Inc. ANSYS. ANSYS, Inc 2014.

[17] EN 15512. Steel static storage system — Adjustable pallet racking systems - Principles for structural design 2009

[18] ABNT NBR ISO 6892:2002. Materiais metálicos - Ensaio de tração à temperatura ambiente. Assoc Bras Normas Técnicas 2002. doi:7704010.

[19] ANSI MH16.1. Specification for the Design, Testing and Utilization of Industrial Steel Storage Racks 2012

[20] Salmon, Charles; Johnson J. STEEL STRUCTURES: Design an Behavior. Emphasizing load and resistance factor design. 5th ed. 2008

[21] Freitas AMS, Souza FT, Freitas MSR. Analysis and behavior of steel storage drive-in racks Thin-Walled Struct 2010;48:110-7. doi:10.1016/j.tws.2009.09.003. 